\title{
Conflicto y cambio en el tercer sector. Carisma, control externo y heterogeneidad en las ONGD
}

\author{
Beltrán Roca Martínez \\ Universidad de Cádiz. Departamento de Economía General \\ beltran.roca@uca.es
}

Recibido: 28-06-2012

Aceptado: 29-05-2013

\section{Resumen}

Este artículo explora el papel del conflicto en las culturas y en los modelos de las organizaciones. A partir del estudio de tres ONGD implantadas en Andalucía - Madre Coraje, Paz y Solidaridad y Manos Unidas-, se analizan cómo los episodios de crisis interna, lejos de destruir a las asociaciones, pueden tener un resultado adaptativo en términos ecológicos a medio plazo, puesto que ayudan a clarificar la identidad de las entidades del tercer sector o a negociar un equilibrio entre diversos sectores y tendencias ideológicas. Se concluye que los episodios de conflicto interno, en estos casos originados por los esfuerzos de entidades externas o figuras carismáticas por controlar las ONGD, frenan y modelan la forma en que las presiones del contexto de la cooperación dirigen a las ONGD hacia la burocratización y la profesionalización.

Palabras clave: organizaciones no gubernamentales; sector no lucrativo; isomorfismo; organizaciones; cultura organizativa; España; gestor de conflictos.

\section{Abstract. Conflict and change in non-profits: Charisma, external control and heterogeneity in development $N G O$ s}

This article explores the role of conflict in organisational cultures and models. Based on the study of three development NGOs located in Andalusia-Madre Coraje, Paz y Solidaridad, and Manos Unidas - it analyses how episodes of internal crisis, far from threatening the survival of organisations, can, in the mid term, have an adaptive effect in ecological terms by clarifying organisational identity or negotiating an equilibrium between different sectors and organisational trends. It concludes that internal conflict, in these cases originating from the attempt of external organisations or charismatic figures to control the DNGO, has the effect of restraining and shaping the way in which environmental factors drive development organisations towards bureaucracy and professionalism.

Keywords: Non-governmental organisations; third sector; isomorphism; organisations; organisational culture; Spain; conflict management. 


\section{Sumario}

1. Introducción

2. Poniendo freno a la profesionalización. Liderazgo carismático en una ONGD andaluza

3. El control sindical sobre la Fundación Paz y Solidaridad
4. El control de la Iglesia católica sobre Manos Unidas

5. Conclusiones: poder, conflicto, isomorfismo y diversidad

Referencias bibliográficas

\section{Introducción}

En la historia de las ONGD (organizaciones no gubernamentales de desarrollo), al igual que en la vida de todas las asociaciones, son frecuentes los periodos de crisis interna. Las entidades del tercer sector son escenario de conflictos y luchas entre sectores que compiten, por ejemplo, por controlar los puestos de decisión, marcar una línea estratégica determinada o imponer ciertos planteamientos ideológicos. Las ONGD no son una excepción.

Durante mucho tiempo, las ciencias sociales han considerado el conflicto colectivo como algo negativo y anómalo (Durkheim, 1995; Parsons, 1988). Del mismo modo, en el estudio de las asociaciones, el conflicto ha sido percibido como algo perjudicial para la supervivencia y el desarrollo organizativo (Mayntz, 1996). No obstante, desde nuestra perspectiva, el conflicto puede favorecer la clarificación de posiciones e intereses dentro de las entidades del tercer sector. Asimismo, permite la adaptación al entorno, utilizando, en términos ecológicos o en otros términos, una reorientación más eficaz de planteamientos, estrategias y tácticas. El conflicto interno no debe verse como una amenaza para la supervivencia de las asociaciones, sino como episodios con un enorme potencial para definir la identidad y la cultura organizacional. Sin embargo, en la bibliografía sobre entidades del tercer sector, siempre se obvia el carácter conflictivo de la cultura, pues se presupone que ésta otorga unidad, armonía e integración en éstas (Schein, 1986; Hofstede, 1999; Scott, 2001; Aguirre, 2004).

El conflicto tiene que ver con las dinámicas internas y externas de las organizaciones. Robert Michels (1996) formuló, a principios del siglo pasado, la ley de hierro de la oligarquía, según la cual las asociaciones modernas tienden a perder su carácter democrático para ser controladas por una minoría. Según Michels, la creciente diferenciación de órganos y funciones que conlleva el desarrollo de toda organización favorece la concentración de poder en un número reducido de comisiones. El crecimiento asociativo, además, provoca la delegación en funcionarios asalariados y la aparición de liderazgos profesionales. Todas las entidades del tercer sector evolucionarían en su desarrollo hacia un mismo modelo. Años más tarde, en esa misma línea, DiMaggio y Powell (1983) propusieron, en un influyente artículo, la hipótesis de la «jaula de hierro», según la cual las organizaciones tienden hacia el «isomorfismo estructural», es decir, a adoptar estructuras similares, debido a procesos mimé- 
ticos, coercitivos y normativos influidos por su entorno. Otros autores han examinado posteriormente estas tendencias homogeneizadoras y han llegado a conclusiones diferentes. Hambrick et al. (2005), por ejemplo, ilustraron cómo diversas tendencias macroculturales disminuyen, en lugar de aumentar, el isomorfismo en el sector empresarial estadounidense. Igualmente, Leiter (2005) destacó que no es posible reconocer dicha homogeneización en el sector no lucrativo australiano, puesto que resulta tan diverso que tan sólo es posible encontrar isomorfismo dentro de cada industria. Todo depende, concluye, del nivel de interacción entre las organizaciones que lo componen. Recientes investigaciones sobre entidades del tercer sector han puesto el énfasis en su creciente profesionalización (Carmel y Harlock, 2008; Blake, 2012).

En España, al igual que en otros países capitalistas avanzados, las ONGD y el conjunto del tercer sector han experimentado un proceso de profesionalización (EDIS, 2012). Diversos factores, entre los que destaca la orientación de determinadas políticas públicas, han propiciado este proceso. Sin embargo, no todas las organizaciones han recorrido las mismas líneas de cambio institucional. El objetivo de este artículo es analizar cómo las presiones internas y externas que inciden en las ONGD se traducen en conflictos y, a través de éstos, dichas asociaciones modelan sus estilos organizativos, sus prácticas y sus discursos.

En efecto, desde nuestro punto de vista, es imprescindible prestar atención al conflicto para analizar el desarrollo organizativo. Estudiar el conflicto, sin embargo, plantea serios problemas éticos y prácticos para los investigadores sociales. Por un lado, a las personas nos incomoda explicar enfrentamientos de los que hemos sido testigos o protagonistas. Hay fuertes intereses y sentimientos que juegan a favor del silencio. Por otro lado, los responsables de las entidades del tercer sector tienden a proyectar hacia el exterior una imagen coherente y unida de sus asociaciones. Si el investigador supera dichos escollos, tendrá acceso a una información muy valiosa para analizar por qué una organización es como es.

Además de la perspectiva conflictual, consideramos que, para el estudio de las organizaciones, es indispensable adoptar una perspectiva diacrónica. Sin conocer la historia de una organización, no es posible alcanzar una explicación satisfactoria de su estructura ni de su funcionamiento, pero para acceder a los conflictos y a la historia de las organizaciones, es preciso adoptar una determinada estrategia de investigación. Para documentar y describir con detalle el funcionamiento de una organización, es necesaria una metodología que permita situarse cerca de aquellos a los que se pretende estudiar. La etnografía es un método de investigación particularmente valioso para el estudio de las organizaciones. En particular, nos permite conocer mejor que cualquier otro método, y de primera mano, la articulación entre los aspectos formales e informales de las estructuras organizativas, las rutinas y los rituales cotidianos, la influencia de los contextos y los significados que los participantes atribuyen a sus acciones. Problematiza, además, los modos en que los individuos y los grupos constituyen e interpretan las organizaciones y las sociedades a partir de sus interacciones cotidianas (Schwartzman, 1993; Rawls, 2008). 
La información de este trabajo procede de una investigación antropológica ${ }^{1}$ sobre las ONGD en Andalucía, en la que se combinaron técnicas etnográficas (observación participante durante varios meses en cuatro organizaciones y entrevistas semiestructuradas a 55 informantes) con el análisis de fuentes documentales. Para este artículo, hemos seleccionado tres ONGD con implantación en Andalucía: la Madre Coraje, Paz y Solidaridad y Manos Unidas. Han sido elegidas por diversos motivos: en primer lugar, la historia de estas organizaciones ha estado jalonada por conflictos internos de mayor o menor intensidad, que han modelado sus estructuras organizativas; en segundo lugar, entre ellas existen suficientes diferencias -ideológicas, de tamaño, de edad, de base social, programáticas y estructurales - como para representar de manera teórica al conjunto del sector. Reconstruyendo la historia de dichas organizaciones y accediendo a su vida interna a través de técnicas etnográficas, trataremos de averiguar el papel de los conflictos en las dinámicas organizativas.

\section{Poniendo freno a la profesionalización. Liderazgo carismático en una ONGD andaluza}

Los orígenes de la Madre Coraje están ligados a la vida de su presidente y fundador, Antonio Gómez. La ONG se funda formalmente en 1993, dos años después del viaje de Antonio a Perú. Un viaje en el que aprendió, según él, el significado de la solidaridad. En 1991, fue invitado por la UNESCO para asesorar dos astilleros del Perú. Allí quedó profundamente afectado por las desigualdades y las injusticias que presenció. A partir de ahí, comenzó una transformación personal, lo que él llama la visión. Antonio regresó a España y decidió hacer algo para ayudar. A los pocos meses, ya estaba constituyendo una ONG.

En un primer momento, reunió a una serie de padres y madres de alumnos de un colegio jerezano para realizar una colecta y enviar ayuda a Perú. Posteriormente, decidieron dar continuidad al trabajo solidario. A los pocos meses, el Ayuntamiento de la localidad les cedió un pequeño espacio dentro de una bodega muy céntrica en desuso. Allí almacenarían los alimentos y el material recogido. A los tres meses, cuenta Antonio, un concejal visitó las instalaciones y se llevó las manos a la cabeza: tenían tanto material que habían ocupado toda la bodega. Posteriormente, tomaron una bodega colindante para almacenar aceite, pues en ella había dos enormes depósitos de vino en desuso. Desde Perú, les habían comunicado la necesidad de jabón para la higiene de los niños y a Antonio se le había ocurrido que podrían hacerlo ellos mismos a partir de aceite usado. Una vez decididos a organizar una asociación, se plantearon el dilema de ponerle un nombre. En Perú, Antonio había oído hablar mucho de una tal

1. Este artículo forma parte de mi investigación doctoral, Anónimo, llevada a cabo entre 2004 y 2008 en el Departamento de Antropología Social de la Universidad de Sevilla y financiada por el programa de Formación del Profesorado Universitario del Ministerio de Educación y Ciencia. 
Maria Elena Moyano, a la que llamaban «Madre Coraje», que lo había dado todo por los sectores más desfavorecidos de su país, y decidieron nombrarla así.

La primera delegación de la Madre Coraje fuera de Jerez se creó en Huelva, a partir de un contacto personal del presidente. La siguiente delegación sería la de Sevilla. Antonio Gómez tenía unos amigos que habían fundado una pequeña ONG de tipo religioso. Por motivos que desconoce, esta organización desapareció. Antonio supo aprovechar la ocasión y les propuso crear una delegación de Madre Coraje. Posteriormente, se implantó en la zona de la Bahía de Cádiz —Cádiz, El Puerto de Santa María y Puerto Real—y, por último, en Málaga. En los últimos años, Madre Coraje se está tratando de expandir por Andalucía oriental y por el resto del Estado español. A través de conocidos y familiares, ha llegado a formar pequeños grupos en Madrid, Pamplona, Oviedo, Toledo y Valladolid. En Andalucía oriental, han constituido «delegaciones en formación» (como ellos las llaman) en Córdoba y Granada. La expansión territorial de Madre Coraje se realiza principalmente a través de redes sociales de amistad y parentesco preexistentes, es decir, a través de amigos - y amigos de amigos- y familiares de la figura central o de sus seguidores más próximos. De este modo, la centralidad de Antonio se ve reforzada. Utilizar vínculos personales suyos o de alguno de los miembros más cercanos de la propia asociación garantiza cierta lealtad.

El crecimiento de Madre Coraje no ha consistido exclusivamente en la apertura de delegaciones a lo largo del territorio andaluz, sino también en el incremento en el número de participantes (socios, voluntarios y trabajadores). Paralelamente, se ha producido una diversificación de las actividades realizadas. El pequeño grupo de ciudadanos que recogía alimentos en el aula del colegio se convirtió, en el año 2000, en una organización que aglutinaba a 543 socios y a unos 700 voluntarios repartidos en siete delegaciones (Madre Coraje, 2000).

En las memorias anuales de la asociación, se encuentran publicados sus balances económicos con un alto grado de detalle. Desde su creación hasta hoy, ha experimentado un rápido crecimiento en el volumen de sus fondos. En 1999, gestionó 546.706 euros (Madre Coraje, 2000) y en el año 2004, 4.282.216 euros (Madre Coraje, 2004). Según la Memoria 2009, Madre Coraje recibió, en el año 2009, un total de 7.411.187 euros (Madre Coraje, 2009: 27).

Conviene destacar que la principal fuente de recursos económicos son las donaciones en especie (45,8\% de los ingresos totales); los ingresos privados monetarios directos - provenientes de las cuotas de los socios, la venta de productos de reciclaje, los apadrinamientos, las donaciones y los patrociniossupusieron tan sólo el 26,5\% (Madre Coraje, 2009: 29). Las delegaciones y los centros de colaboración de la asociación reciben constantemente una ingente cantidad y multiplicidad de materiales usados (medicamentos, ropa, aceite, radiografías, libros, etc.). Desde el área de Ayuda Humanitaria, estos recursos son enviados directamente a Perú, reciclados o vendidos a empresas para obtener fondos.

En relación con los participantes, Madre Coraje cuenta hoy con 1.274 socios, 974 voluntarios y 35 trabajadores remunerados. Aunque es la ONGD 
con más voluntarios en Andalucía, la figura del socio aún no está plenamente desarrollada. La mayor parte del voluntariado se centra en las actividades de ayuda humanitaria. El perfil del voluntario es el de una mujer, de edad avanzada, ama de casa y católica, aunque varía según el tipo de actividad. El perfil de los socios es similar al de los voluntarios, pues, en gran medida, son las mismas personas.

\section{Contratación de personal: un punto de inflexión hacia la formalización}

En un primer momento, Antonio Gómez estaba en contra de tener personal asalariado. Pensaba que la solidaridad no debía convertirse en el negocio o en el medio de vida de algunas personas. A medida que la asociación fue creciendo, se dio cuenta de que era necesario disponer de gente que se dedicara a determinadas tareas a tiempo completo y de gente cualificada para realizar ciertos trabajos - desde las funciones administrativas hasta el diseño y el seguimiento de proyectos de desarrollo-. Ello requería necesariamente contratar personal.

Hubo un gran cambio. Yo, en un principio, no quería profesionales en Madre Coraje, pero me di cuenta de que, de que desaparece Madre Coraje si no pongo profesionales, puesto que la misma contabilidad yo no podía. Tenía un voluntario y se me iba... Había cosas de que la credibilidad o la transparencia que nosotros normalmente queríamos llevar a cabo pues no la podíamos llevar a cabo si no es con profesionales. (Antonio Gómez, 75 años, ingeniero jubilado, presidente de Madre Coraje, Jerez de la Frontera. Entrevista)

Desde 1998, año en que se contrató a los dos primeros asalariados, hasta 2000, se formalizaron seis contratos fijos y nueve con carácter temporal (tres del total en Perú). Este periodo es clave. Hasta entonces, una buena parte del «voluntariado» estaba formado por objetores de conciencia que realizaban la prestación social sustitutoria al servicio militar obligatorio en la asociación. Había unos 50 objetores en Jerez, unos 20 en Sevilla y algunos en otras delegaciones. Pero llegó el momento en que el servicio militar dejó de ser obligatorio y entonces se comenzó a palpar el miedo a entrar en crisis como consecuencia de la falta de voluntariado.

Las primeras contrataciones no fueron idea del presidente, sino que fueron promovidas por los propios voluntarios que necesitaban un empleo remunerado. Antonio se vio obligado a ofrecerles trabajar para la asociación ante la incapacidad de encontrar sustitutos para las tareas que venían realizando. Una empleada explica la polémica suscitada cuando fue contratada:

Antonio no quería contratar a nadie, eso era claro. Era la asociación pura y no quería contratar a nadie. Al final, se vio obligado por las circunstancias a contratar a gente. $\mathrm{O}$ sea, la idea de asociación de él y del grupo que entonces dirigía, que era un grupo de personas mayores que conformaban la Junta Directiva, que eran de Jerez. Y bueno, cuando me contrataron a mi se lió una en la Junta Directiva que vamos. Lo sé porque a mi me lo han 
contado, fue horroroso, y todavía le tengo que agradecer a uno el hecho de que fuera capaz de ver la necesidad y explicarla. (Verónica, 40 años, empleada, responsable de coordinación territorial de Madre Coraje, Jerez de la Frontera. Entrevista)

La contratación de empleados trajo consigo dos conflictos. En primer lugar, entraron en escena una serie de participantes movidos - aunque no exclusivamente- por un interés salarial, un interés que, en ocasiones, puede entrar en contradicción con los fines de la organización. Verónica, por ejemplo, comentaba que, al principio, por el hecho de recibir remuneración por su trabajo, tenía la sensación de estar «robando dinero a los pobres». En segundo lugar, algunos de los contratados eran, a su vez, profesionales, es decir, "expertos» dispuestos a influir en la organización y a modelarla según las recetas que se prescriben desde los ámbitos técnicos y científicos ${ }^{2}$.

Estos primeros empleados detectaron desde el principio la necesidad de normalizar las actividades mediante la planificación. El rápido crecimiento organizativo y la ampliación de las áreas de actividad hicieron que los participantes viesen necesario formalizar la estructura de la asociación y contar con personal que le prestara dedicación integral. En esta línea, Sergio promovió y lideró la primera planificación estratégica en el año 2000 (aunque acompañado en todo momento por Antonio y Verónica). Con el crecimiento de la asociación, la ausencia de planificación dejaba todas las actividades a merced de la improvisación. Para algunos profesionales, esto implicó que se viniera haciendo un conjunto de actividades incoherentes entre sí y de difícil evaluación. El objetivo de la planificación era desarrollar un diagnóstico de la situación, un esclarecimiento del posicionamiento ideológico, para, a partir de ahí, establecer metas alcanzables. En otras palabras, mejorar el funcionamiento interno de la organización e incrementar la eficacia de sus acciones.

Hasta el año 2000, los primeros profesionales tenían una gran influencia dentro de la asociación. Algunos, como Sergio, llegaron a ser la «mano derecha» de Antonio. Sin embargo, las diferencias ideológicas y estratégicas se fueron haciendo más manifiestas y Antonio aprovechó la reestructuración de la organización para acotar el poder de los trabajadores remunerados.

Sí, es cierto que en ese momento había una cierta apertura a que los «profesionales", a que los pocos "profesionales» que en ese momento trabajábamos, pues asesoráramos y estábamos muy cerca de Antonio. Es ese momento en el que Antonio dice que los «profesionales» llevábamos un poco la voz cantante. Eso se da antes de la planificación, durante la planificación y un poquito después de la planificación, un año después de la planificación o así. (Sergio, 38 años, empleado y responsable del área de proyectos de Madre Coraje, Jerez de la Frontera. Entrevista)

2. De todos modos, hay que señalar que, a pesar de las tensiones internas, Madre Coraje es de las pocas ONGD implantadas en Andalucía que cuenta con comité de empresa y un acuerdo interno que regula las relaciones laborales. 
Precisamente en ese momento se creó el puesto de gerente, cuya constitución y proceso de selección no estuvo exento de polémica, pues, para unos, el papel debía ayudar a consolidar y profundizar el proceso de profesionalización y, para otros, debía ser la herramienta para mantener al margen a los empleados de los órganos de decisión ${ }^{3}$.

\section{Autonomía o centralización}

La expansión de una organización plantea el problema de la coordinación entre las distintas estructuras territoriales. A lo largo de los primeros años, Madre Coraje era un archipiélago de comités de solidaridad con Perú independientes y encabezados por una asociación más importante ubicada en Jerez. En el año 2002, se abrió un proceso de negociación para establecer las condiciones en que se trataría de unificar y coordinar mejor las distintas delegaciones de la asociación. En ese proceso de negociación, se distinguía claramente la posición de Jerez de la Frontera y la que mostraban las delegaciones colindantes, que optaban por una alta centralización (de recursos, de decisiones y de líneas de acción), y la de la delegación de Sevilla, que luchaba «a capa y espada» por preservar su autonomía. "Nosotros defendimos a toda costa la independencia de Sevilla», me comentaba un voluntario sevillano que estuvo presente en las negociaciones.

Autonomía o centralización. Había dos sistemas de organización completamente distintos y había que optar por uno de los dos. Tenías a Sevilla, que quería plena autonomía y tenías Cádiz, Puerto Real y tal que querían centralización. Entonces vimos que la autonomía tenía una ventaja, da responsabilidad, capacidad de iniciativa, sin embargo, el problema que tiene es que es muy difícil el trabajo en equipo entre delegaciones. Se hizo un término medio: en el plano financiero, tenéis total autonomía [...] En las delegaciones que están empezando, esto nos ha traído problemas. Por ejemplo Granada, se le dio autonomía y el primer año dio pérdidas y tuvo la central que pagarles. (Antonio Gómez, 75 años, ingeniero jubilado, presidente de Madre Coraje, Jerez de la Frontera. Entrevista)

Uno de los puntos más polémicos fue la administración de los recursos monetarios y de los socios. Sevilla consiguió que las delegaciones preservaran la autonomía financiera y la gestión de los socios. Respecto al número de votos de cada delegación, en cambio, no logró imponer que fuera proporcional,

3. Antonio no comparte todas las percepciones de los profesionales, particularmente su énfasis en la planificación. «Están cuadriculados», me comentaba. «Es decir, ellos hacen la planificación y lo que no esté en la planificación no se puede hacer. Yo siempre les cuento, que les da mucha rabia [ríe], la historia de aquel planificador que fue al monte, no sé si tú lo sabes, a coger setas y en esto, cogiendo setas, se encontró con un billete de 100 euros. Y entonces se queda pensando y dice: " $\mathrm{iNo}$, cuando setas, setas, y cuando euros, euros! Yo solamente cojo setas". Esto es una caricatura del planificador, que el problema que tiene, también tiene muchísimas ventajas, es que confunde el fin con los medios.» 
según Antonio, a la «importancia de la ciudad». «Nos costaba mucho que entendiera Sevilla que, aunque fuera más grande, eran para nosotros igual de importantes que Puerto Real, y eso no lo entendían", aclara Antonio Gómez. En la Junta Directiva, cada delegado es un voto. En cambio, en la Asamblea General, los votos son proporcionales al número de socios, pero hay un tope que afecta a las delegaciones grandes como Sevilla: cada delegación dispone de dos «compromisarios» (representantes con voto) de partida, más uno adicional por cada cincuenta socios, hasta llegar a un máximo de diez compromisarios por delegación. Esta negociación se plasmó en los estatutos del año 2002.

\section{El conflicto entre áreas}

Las actividades de Madre Coraje se realizan en tres áreas: Ayuda Humanitaria, Proyectos y Educación para el Desarrollo.

La ayuda humanitaria es la primera actividad que realiza la ONGD. Es el área más antigua, la que la justifica y a la que se supeditan y para la que se adecuan las demás. En los comienzos de la asociación, la división de tareas era algo informal, y los pocos voluntarios que había se encargaban de hacer un poco de todo. Con el tiempo, se fue acentuando la división del trabajo y se empezaron a contratar empleados, tanto para tareas de mantenimiento y transporte, como para la gestión y coordinación. El funcionamiento se ha ido formalizando, especialmente tras la contratación de Magdalena, actualmente responsable del área. En la actualidad, ésta se divide en diferentes grupos de trabajo, cada uno con unas tareas bien definidas. Además, la actividad de reciclaje y la de ayuda humanitaria se encuentran diferenciadas. El reciclaje incluye: recogida y envío de ropa; fabricación de jabón; recogida de aceite usado, de alimentos, de medicinas; clasificación de libros, de material sanitario, de juguetes; mantenimiento y almacén.

El área de Proyectos se encarga de realizar las tareas de desarrollo en Perú. Su actuación está altamente profesionalizada. La mayor parte de sus integrantes son empleados con una cualificación en elaboración y gestión de proyectos de cooperación para el desarrollo. Sergio, responsable de dicha actividad, comenta que "por cómo trabajamos, es un área eminentemente técnica o profesional». La participación del voluntariado y el conocimiento sobre las actividades es muy reducida.

El área de Educación, por otro lado, es la más reciente. Se creó en el año 1998 con la contratación de Jacinto, en respuesta a una demanda de los participantes de la asociación de llevar a cabo actividades educativas y de «sensibilización", al igual que estaban haciendo el resto de ONGD. En ella, hay, en la actualidad, cuatro personas contratadas y un pequeño grupo de voluntarios.

Las relaciones entre las tres áreas han sido muy conflictivas. En un momento dado, surgieron roces personales entre los responsables de Ayuda Humanitaria y los de Proyectos. La solución que adoptó el presidente fue aislar el funcionamiento de cada una. El resultado ha sido que los planteamientos sobre la ayuda y el «desarrollo» son distintos entre los participantes de cada área (aparte 
de la falta de coherencia y coordinación). Además, existe competencia entre las áreas por los recursos de la asociación y por representarla simbólicamente. Para algunos, desde la dirección, hay una clara preferencia por Ayuda Humanitaria:

El área de Proyectos y el área de Ayuda Humanitaria están compitiendo por recursos de la asociación..., y están compitiendo de alguna manera por la imagen de la asociación. La imagen tradicional de Madre Coraje es una imagen ligada a la Ayuda Humanitaria, y sigue siéndolo, y el área de Proyectos pretende que su trabajo también forme parte de esa imagen. (Sergio, 38 años, empleado y responsable del área de Proyectos, Jerez de la Frontera. Entrevista)

Otros, como el presidente, no están de acuerdo con eso. Aducen que las cifras que manejan los que argumentan la primacía de Ayuda Humanitaria están manipuladas, al contabilizar el valor dinerario de las donaciones en especie, aunque reconocen que Ayuda Humanitaria aporta la mayor parte de fondos y de voluntarios a la asociación, y que la combinación de «reciclaje y solidaridad» es una fuerte seña de identidad de Madre Coraje.

\section{Concepciones en disputa}

Los conflictos internos de Madre Coraje están conectados con la disputa entre distintas concepciones del «desarrollo» y la ayuda. Una disputa que enfrenta a las tres áreas entre sí y a dos sectores de la organización: Antonio y sus seguidores, por un lado, y un pequeño grupo de profesionales, por otro.

Si bien existe un consenso formal en torno al discurso del «desarrollo humano y sostenible», mientras entre el grueso del voluntariado prevalecen unas concepciones «asistencialistas» de la ayuda, un pequeño grupo la entiende como «cooperación». Entre la mayoría de los voluntarios del área de Ayuda Humanitaria, subyace la idea de que la escasez de recursos es la causa de la pobreza, por lo que la solución pasa por enviar recursos en especie, como alimentos, medicamentos, ropa, libros, jabón o suero glucosalino. Las otras dos áreas, integradas en su mayoría por profesionales, optan de manera más decidida por un «desarrollo humano y sostenible», así como por una cooperación entendida como trabajo conjunto. Desde el área de Educación, además, se insiste en la importancia de la enseñanza y la sensibilización en el Norte acerca de la transformación de las estructuras mundiales.

Antonio Gómez utiliza los principios de la organización para dotarla de una identidad que la diferencie de otras ONGD y que sirva para combatir las divisiones internas. De este modo, se refuerza su autoridad, puesto que él encarna dichos principios. Ha promovido la creación de muchos documentos en los que se recogen y se difunden las bases de Madre Coraje. Quizá el documento El Sindrome de la Misión sea el que mejor ejemplifique el propósito del fundador. Este texto, fechado el 20 de agosto de 2005, fue enviado por Antonio Gómez a todas las áreas, servicios y delegaciones de Madre Coraje. El «síndrome de la misión» no es otra cosa que la confusión entre los fines y los medios en la que 
caen - desde la perspectiva de Antonio- algunos empleados y profesionales. No todos los participantes de Madre Coraje, sin embargo, están igualmente identificados con estos principios, ni todos siguen a Antonio Gómez con el mismo fervor, ni todos defienden unas mismas concepciones del desarrollo. Antonio sostiene que el conflicto no es ideológico, sino "etimológico». El problema, a su entender, es la obsesión de los profesionales por la planificación, que enturbia el funcionamiento armonioso entre las distintas áreas y los participantes en la organización.

\section{El control sindical sobre la Fundación Paz y Solidaridad}

La Fundación Paz y Solidaridad es una de las herramientas del sindicato Comisiones Obreras para hacer frente a los principales desafíos que la globalización capitalista plantea al mundo del trabajo, como la inmigración y la deslocalización de las empresas. Esta ONGD constituye un instrumento para desarrollar su política internacional, encaminada a contrarrestar los efectos negativos de la globalización estableciendo alianzas con organizaciones sindicales de otros países.

En Andalucía, se creó en 1992 como delegación de la Fundación Paz y Solidaridad Serafín Aliaga, de ámbito estatal y perteneciente a Comisiones Obreras, siguiendo la decisión tomada en el congreso confederal de este sindicato, celebrado en 1990, en el cual se alcanzó el acuerdo de constituir la Fundación en dicha comunidad. En el núcleo inicial de Sevilla, se encontraban algunos profesores afiliados a la Federación de Enseñanza, una abogada del sindicato que había hecho un curso de la AECI sobre mujeres y desarrollo, amigos de afiliados que estaban desempleados y una persona que trabajaba en el gabinete técnico del sindicato. Entre ellos, destacaba Mercedes Guerrero, que llegaría a ser, por aquel entonces, vicepresidenta de la Coordinadora Andaluza de ONGD. «En Andalucía esto siempre surge del grupo de personas que se identifican más con estas cosas», afirma el actual presidente, Antonio Andrade. «Los temas de cooperación siempre surgieron de los propios afiliados o de los dirigentes sindicales que eran más sensibles a esos temas", añade.

Desde un primer momento, la dirección del sindicato se esforzó por dotar de estructura a la iniciativa. Se creó una directiva, con Juan José García, de la Federación de Enseñanza, a la cabeza:

Básicamente, más o menos existía una estructura similar a lo que ahora es el patronato, pero más reducido. O sea, no era una fundación, era más una asociación. Entonces existía su presidente, su director, dependía de una secretaría de Comisiones Obreras de Andalucía y después teníamos algunas personas que participaban de forma voluntaria. Y después, bueno, había otras personas de algunos territorios de Comisiones Obreras de Andalucía y de algunas federaciones que participaban. Existía un órgano más reducido, más reducido, efectivamente, que ya cuando se toma la determinación de hacer la Fundación. (Antonio Andrade, 60 años, director de la Fundación Paz y Solidaridad de Andalucía. Entrevista) 
Además, se marcó el objetivo de ampliar la ONGD al resto de provincias andaluzas. "También en esa época hubo un primer taller en Viznar, en Grana$\mathrm{da}$, una cosa de multiplicación, de invitar a gente del sindicato para implicar a la base de la Fundación: gente de inmigración de Málaga, un hombre de Argelia que ya no sigue en la Fundación", comenta Roberto, el primer empleado.

La ONGD se constituyó legalmente como fundación en abril de 1995. El patronato estaba compuesto por un conjunto de militantes, en su mayoría del sector «oficialista», de Comisiones: Juan José García Vera (presidente), Francisco Cordero López (director), Jaime Montes Muñoz (secretario), Francisco Carbonero Contador (tesorero), y Francisco Rodríguez Martínez, José Conde Villanuevas, María Luisa Madrera Pareja y Marcos González Sedano (vocales). Al poco tiempo, Francisco Cordero abandonó la ONGD para implicarse desde entonces en otras iniciativas sociales al margen del sindicato. Se convocó una reunión con toda la gente que trabajaba voluntariamente en la Fundación y se decidió que hacia falta un coordinador con un contrato de ocho horas semanales. Ofrecieron la plaza a Roberto, uno de los voluntarios, que provenía de la Federación de Enseñanza y formaba parte del núcleo inicial.

En aquel momento, cuando la ejecutiva del sindicato eligió la composición del patronato, la ONGD experimentó una dura crisis interna. Muchas de las personas que habían formado parte del núcleo inicial sentían que habían perdido capacidad de decisión, de control sobre la Fundación, y decidieron abandonarla.

Ninguno estaba involucrado políticamente en ningún movimiento. Era un poco light. Era un grupo muy democrático y muy protegido de la dureza de la vida de Comisiones Obreras. Éramos como cualquier otra ONG. La cooperación que hacíamos era clásica, por decirlo de alguna manera; que no era cooperación sindical. Era un poco como una burbuja, y lo que pasó era lógico: que esas personas eran ajenas a las directrices políticas de Comisiones. (Luz, 43 años, voluntaria del núcleo inicial de Paz y Solidaridad, Sevilla. Conversación informal)

La crisis de Paz y Solidaridad se saldó con la pérdida de toda su base de voluntariado en Andalucía, que o bien abandonó el compromiso social o bien se implicó en otro tipo de organizaciones de la sociedad civil. Desde 1997, no hay voluntarios en la ONGD.

En marzo de 1997, se renovó el patronato de Paz y Solidaridad y se constituyó en Andalucía con personalidad jurídica propia. Antonio Andrade Romero fue nombrado presidente. Los dirigentes de las diversas estructuras sectoriales y territoriales de Comisiones Obreras en Andalucía figuran en numerosos cargos y órganos. Para entender la estructuración y el desarrollo de la ONGD, debemos atender, además, a las relaciones entre los sectores enfrentados en el interior del sindicato. Comisiones Obreras de Andalucía ha estado siempre dominado por el sector "oficialista», que ha puesto a personas de su confianza para gestionar Paz y Solidaridad en la comunidad autónoma. Sin embargo, se ha dado la circunstancia de que la unión provincial de Comisiones Obreras de 
Sevilla ha estado dominada por el sector «crítico» hasta mediados de 2008, en el que, en un congreso de dicha rama del sindicato, los «oficialistas» lograron imponerse. Durante años, desde la unión provincial, no se habían preocupado por fomentar a la Fundación, pero, a partir de aquella fecha, las cosas cambiaron. A nivel autonómico, en 1992, el sector "oficialista» ${ }^{4}$ consiguió que Julio Ruiz fuera elegido secretario general de Comisiones Obreras de Andalucía 5 y Antonio pasó a formar parte de la dirección general del sindicato en dicha comunidad, al mismo tiempo que continuó en el comité de empresa de un centro de trabajo que pertenecía al Banco Central. Dicho centro de trabajo se había puesto a la venta y había sido comprada por la multinacional sueca Boliden. Tras la catástrofe ecológica, con la ruptura de la balsa, la empresa cerró y prejubilaron a los empleados mayores de 52 años, entre ellos, Antonio Andrade.

Por esa época, la Fundación Paz y Solidaridad ya funcionaba en Andalucía como delegación de la fundación estatal Serafín Aliaga, pero, en 1995, se decidió crearla a nivel andaluz y él fue elegido presidente, a propuesta de Julio Ruiz, secretario general del sindicato en Andalucía. Desde entonces, Antonio Andrade se dedica «en cuerpo y alma» al trabajo de cooperación.

El patronato de 1997 prosiguió aproximadamente con la misma composición hasta que, en el año 2004, Alfonso Martínez Foronda, profesor de historia de la Universidad de Granada (y liberado sindical) fue elegido, en un congreso confederal, secretario de Fundaciones y Estudios y presidente de las dos fundaciones de Comisiones Obreras de Andalucía: la Fundación Archivos Históricos y la Fundación Paz y Solidaridad. Desde aquel momento, Antonio Andrade ejerce como director.

En el trabajo cotidiano de la ONGD, la supervisión directa del director constituye un papel importante. Existe, además, cierta división de tareas: tres empleados trabajan en actividades de sensibilización; una empleada se encarga de la comunicación y la imagen; otra destina la mitad de su tiempo a tareas administrativas, y otra, a la gestión de proyectos de cooperación. El resto de trabajadores se dedica en exclusividad a la gestión de proyectos de cooperación y están divididos por áreas: uno se encarga de África y el Mediterráneo; otro, de Centroamérica, y otro, de Sudamérica. Aunque, en la práctica, «se borran un poco las fronteras. Todo lo mezclamos un poco", reconoce Roberto.

Además, hay reuniones de todo el equipo una vez al mes en Sevilla. En ellas, cada trabajador expone en qué está trabajando. Al igual que en otras ONGD estudiadas, los empleados de Paz y Solidaridad de Andalucía reclaman que los directivos presten mayor atención a la planificación estratégica. Así lo expresaba un informante que había trabajado para la Fundación:

4. El sindicato Comisiones Obreras se encuentra dividido internamente entre varias facciones que pugnan por la hegemonía. Los dos principales sectores son los "críticos», partidarios de radicalizar el sindicato, y los «oficialistas», más partidarios de la negociación y la inserción en los órganos del Estado.

5. Ver, por ejemplo, el artículo sobre la reelección de Julio Ruiz en el año 2000: «El enfrentamiento interno marca el octavo congreso regional de CC. OO.», El Pais (17 de julio de 2000). 
La planificación es una cosa de la que nosotros como técnicos siempre nos hemos quejado. La falta de planificación. Siempre nos basamos un poco en lo que es la reacción a circunstancias, posibilidades..., más que hacer una anticipación. (Julieta, 45 años, antigua empleada de la Fundación Paz y Solidaridad de Andalucía. Conversación informal)

A nivel estatal, Paz y Solidaridad de Andalucía forma parte de la red Paz y Solidaridad Serafín Aliaga, que es igualmente una fundación vinculada a Comisiones Obreras. Dicha fundación está integrada por un patronato que es elegido por la Comisión Ejecutiva del sindicato. El patronato, integrado por un número que puede oscilar entre 9 y 21 personas, es el órgano de gobierno, representación y administración de la ONGD. La red estatal contaba, en 2006, con 78 empleados, 7 de ellos en Andalucía. Diferencian entre «asalariados» y «liberados». Mientras los primeros son personas contratadas con fondos de la ONGD, los segundos son personas liberadas por sus empresas que realizan su labor sindical en el seno de la Fundación. Según la Memoria Anual 2009 (Paz y Solidaridad, 2009), la ONGD recibió, en el año 2006, un total de 11.525.448,18 euros en todo el Estado español. El 89,50\% de los ingresos contabilizados en el 2009 procedieron de fuentes públicas y sólo el 10,50\%, de financiadores privados. La dependencia respecto a las instituciones públicas es evidente. En los últimos años, se ha producido un aumento en el total de recursos financieros gestionados por la ONGD.

Una de las características de la Fundación es su reinterpretación del discurso del «desarrollo». Se apropian del discurso del «desarrollo» y lo resignifican desde el ámbito del sindicalismo: el poder de los sindicatos como motor e indicador del nivel de "desarrollo" de un territorio. "Sin respeto por los derechos laborales fundamentales no puede haber desarrollo", han sugerido Núñez y Santos (2004: 178), a lo que añaden que «está más que demostrado que unos sindicatos fuertes, representativos, democráticos y autónomos, juegan un papel fundamental en el desarrollo económico y social en sus respectivos países». Siguiendo la distinción entre crecimiento y desarrollo establecida formalmente por el PNUD, subrayan que «el concepto de desarrollo incluye obligatoriamente la equidad, que debe acompañar al proceso de crecimiento económico sostenible» (Nuñez y Santos, 2004: 179). El desarrollo consiste, por tanto, en que los resultados del crecimiento económico sean distribuidos equitativamente, entre otras cosas. Así lo expresa Antonio Andrade:

Lo cierto es que es un elemento nuevo de la cooperación, es decir, los pueblos se «desarrollan» también y fundamentalmente en la medida en que se desarrolla la sociedad en su conjunto y conjuntamente los trabajadores. A medida que una sociedad avance en mejoras económicas, en sistemas democráticos, en sistemas de participación, eso es «desarrollo». Y en la medida en que, dentro de ese contexto, los trabajadores mejoren sus condiciones económicas, de vida, laborales, culturales, eso es «desarrollo». (Antonio Andrade, 60 años, director de la Fundación Paz y Solidaridad. Entrevista) 
A partir del año 2001, la ONGD comienza a especializarse en «cooperación sindical».

\section{El control de la Iglesia católica sobre Manos Unidas}

En julio de 1960, la FAO lanzó su primera campaña mundial contra el hambre. Para llevarla a cabo, solicitó la ayuda de las ONGD. Cinco años antes, la Unión Mundial de Organizaciones Femeninas Católicas (UMOFC) se había pronunciado sobre la necesidad de emprender acciones contra el hambre en el mundo (Vidal, 1997). Así, en 1960, las Mujeres de Acción Católica, con Mary Salas Larrazábal a la cabeza, respondieron a la llamada y recogieron 500.000 pesetas (unos 3.000 euros) en el Estado español. Mujeres de Acción Católica era una organización femenina dentro de la Federación de Movimientos de Acción Católica Española, una asociación de seglares que trabajaba junto a la jerarquía de la Iglesia católica por la evangelización.

Por el éxito de la colecta y la gravedad de la situación mundial, estas mujeres, bajo el liderazgo de Josefina Martín Sanpedro, fueron repitiendo la campaña cada año. En 1978, decidieron constituirse legalmente como asociación con personalidad jurídica propia y eligieron a Pilar Villar de Días como dirigente. Desecharon el nombre Campaña contra el Hambre, porque hacía referencia a una acción en la que participaban muchos actores, y optaron por el de Manos Unidas. La Iglesia les apoyó, aparte de con la colecta, facilitándoles espacio en las diócesis. En la década de 1980, se fue consolidando la organización y, en 1981, aprobaron los primeros estatutos.

En la década de 1990, al igual que otras ONGD, Manos Unidas experimentó un rápido crecimiento en términos de voluntarios, empleados y recursos financieros. Gente nueva empezó a adherirse a la asociación. "Marchaba perfectamente», explica Gema, una antigua delegada de Cádiz, «había mucho personal muy preparado», añade. Este crecimiento conllevaba cambios significativos en la función, en la estructura, en los estilos de gestión y discursos sobre el «desarrollo» y la solidaridad por parte de la organización y sus participantes. La presidenta en el periodo 1990-1997, Ana de Felipe, explicaba de este modo las transformaciones en el modelo organizativo que tuvieron que promover para hacer frente a la nueva realidad:

Entre otras cosas, en esta etapa, se reforzó en gran medida los recursos humanos, tanto voluntarios como contratados, y se mejoró su formación. [...] Al volverse más complejo y específico el trabajo y ser muy difícil encontrar voluntarios que lo asumieran, por la disponibilidad y por la preparación concreta que requería esa tarea, se contrataron profesionales que lo pudieran llevar a cabo. (Ana de Felipe Bonete, en Escartín, 2009: 106-107)

La tensión entre el carácter eclesial de la organización y la secularización de la «ayuda al desarrollo» que se había extendido en el conjunto de las ONGD no había brotado aún a la superficie de Manos Unidas, pues todas las dirigentes habían desarrollado su labor en estrecha colaboración con la jerarquía 
eclesiástica. Con Ana de Felipe Boente, presidenta en el periodo 1990-1997, esta tensión dio lugar a una profunda crisis interna. Durante su mandato, la Conferencia Episcopal solicitó a Manos Unidas parte de sus fondos para proyectos de evangelización. La presidenta y la asamblea de delegados rechazaron la petición de los obispos y les acusaron de intromisión. Desde ese momento, se empezó a materializar un clima de desconfianza entre la jerarquía eclesiástica y la dirección de la ONGD.

En 1997, bajo el mandato de Consuelo Lobo, comenzaron las negociaciones para renovar los estatutos de la asociación. Los obispos querían que la ONGD clarificara su identidad como organización de la Iglesia católica, la formación religiosa de sus miembros, la intervención de la Conferencia Episcopal en la elección de delegados y la participación de los empleados. A principios de 2000, constituyeron un comité para elaborar los estatutos en el que todas las partes estuvieron presentes. Estos estatutos fueron ratificados luego por la asamblea de Manos Unidas. De acuerdo con ellos, posteriormente, la Conferencia Episcopal debía aprobar o rechazar los nuevos, pero, en lugar de eso, cambiaron parte de su contenido. El artículo 2.3 decía antes: «Por ser Manos Unidas una organización de voluntarios, los cargos de coordinador de área y departamento — que son puestos de gestión y no de gobierno- serán ocupados por los voluntarios "adecuados"; sólo si no es posible, en opinión del Comité Permanente, podrán ser ocupados por personal contratado»; y los obispos suprimieron la segunda parte, que consideraba la posibilidad de que profesionales contratados ocuparan cargos de responsabilidad si era necesario. La intromisión de la Iglesia provocó la dimisión de parte de la directiva, de muchos voluntarios y de la mayor parte de las personas contratadas. Como algunos argumentaron:

La Conferencia Episcopal Española ha modificado esencialmente la propuesta presentada por Manos Unidas. Ésta había sido elaborada con la participación de la Conferencia Episcopal y aprobada por dos tercios de los delegados de la Asamblea y por el Comité directivo de Manos Unidas (ambos compuestos en su totalidad por voluntarios), saliendo exitosas las instrucciones de la Conferencia Episcopal. Han decidido, por tanto, en contra de la mayoría de las voluntades de la organización, socavando la autoridad de los órganos de gobierno. (Editorial, Revista de Fomento Social, 2000: 315)

Aquel año debían elegir una nueva presidenta. De las 11 candidatas, la Conferencia Episcopal vetó a 5. Después de esa intervención, Consuelo Lobo dimitió. Durante la asamblea, Ana Álvarez fue elegida nueva presidenta, pero con muy poco apoyo: sólo 24 de las 63 delegadas la votaron, pues hubo 31 abstenciones, 2 votos en blanco y el resto votó a favor de otras candidatas. Después de la asamblea, dimitieron 10 delegadas. Declararon que la intromisión de la Conferencia Episcopal había alterado el proceso electoral de la

6. Más adelante, en los estatutos, los obispos clarificaban qué entendían por «adecuado»: católicos practicantes. 
ONGD y que, después de eso, no podían continuar trabajando de la misma manera. Cuando los delegados volvieron a sus delegaciones y contaron lo ocurrido, muchos voluntarios, socios y empleados abandonaron la organización. Varias delegaciones cerraron. Mucha de la gente que salió de Manos Unidas fue a parar a otras ONGD confesionales, como Entreculturas-Fe y Alegría e Intermón Oxfam. Otros, a pesar de su desacuerdo con los obispos, decidieron continuar en la asociación.

Un tercer grupo más conservador estaba de acuerdo con la Conferencia Episcopal. Monseñor Omella y monseñor Asenjo escribieron una carta en nombre de la Conferencia Episcopal dirigida a los miembros de Manos Unidas, con la cual pretendían minimizar los efectos de la crisis y negaban haber solicitado a la dirección de la ONGD dinero para proyectos de evangelización ${ }^{7}$. En ella, argumentaban que disponían de sus propios fondos para esos propósitos — que llamaban «Fondos de Nueva Evangelización»—. Este fondo, sin embargo, fue creado exactamente en 1997, precisamente cuando la Junta de la ONGD supuestamente rechazó usar sus recursos para la evangelización explícita.

La nueva presidenta, en la línea de los obispos, criticaba la «excesiva profesionalización» que la ONGD estaba experimentando, puesto que ello podía poner en peligro el espíritu cristiano de la asociación. Pero lo que estaba en juego no era tanto si contratar o no contratar empleados, sino el control de la Iglesia sobre la ONGD. Por aquel momento, como ya hemos señalado, se estaban produciendo cambios importantes en el discurso del «desarrollo». Intermón Oxfam se había distanciado de la Compañía de Jesús. Y un sector considerable de representantes y empleados de Manos Unidas se esforzaron por separar evangelización de desarrollo. El resultado fue dramático.

En los años sucesivos, la ONGD se recuperó de la crisis conservando la orientación marcada por los obispos. En 2006, fue elegida presidenta Begoña de Burgos López, su mandato daba continuidad a la línea de Ana Álvarez.

A lo largo de los años, el número de participantes ha crecido de forma constante. Hoy en día, cuentan, según su memoria, con 4.500 voluntarios estables, 15.000 colaboradores para la campaña de febrero, alrededor de 100 empleados y 85.575 socios. Aunque cualquiera puede ser voluntario a nivel de base, a la hora de ocupar cargos es imprescindible acreditar la condición católica y contar con el beneplácito tácito de la Conferencia Episcopal. Así lo expresa monseñor Omella en un esclarecedor artículo:

El voluntariado que busca sólo el quehacer y no el ser. Me refiero a aquellos que se acercan a las acciones socio-caritativas de la Iglesia sin «una adhesión explícita de fe» y sin una voluntad abierta a la posibilidad de que el servicio caritativo que prestan, cambie y convierta sus vidas. No sería deseable que este tipo de voluntarios fueran sin más rechazados. Tampoco sería bueno que

7. La carta fue publicada en el número 234 de la revista Alfa y Omega (16 de noviembre de 2000). 
llegaran a predominar, ni que se le encomendaran tareas de dirección, porque probablemente se generaría un déficit de identidad y eclesialidad. (Monseñor Omella) 8

Manos Unidas nace y se desarrolla al amparo de la Iglesia católica. Esta íntima relación, afianzada tras la crisis del año 2000, dota a la ONGD de una serie de características propias, entre ellas, un discurso que combina elementos religiosos y elementos desarrollistas. Como explica Lourdes Azorín, secretaria general de Acción Católica, en un boletín de Manos Unidas: «Desde el primer momento se habló de luchar contra tres hambres: el hambre de pan, el hambre de cultura y el hambre de Dios. Esto supone la promoción integral de la persona» ${ }^{9}$. Se trataba, al fin y al cabo, de dar a los otros aquello de lo que, a su modo de ver, carecían: alimentos, cultura y fe en Dios. Desde entonces, su discurso se ha ido transformando, pero, a diferencia de otras ONGD, el componente religioso ha permanecido fuertemente arraigado. Se dice, por ejemplo, que el crecimiento no debe ser sólo económico y que el crecimiento tiende además hacia Dios: «De la misma manera que la creación entera está ordenada a su Creador, la criatura espiritual está obligada a orientar espontáneamente su vida hacia Dios, verdad primera y bien soberano». En esto consiste, para la Iglesia, el «desarrollo integral», un proceso que no es exclusivamente técnico.

No obstante, en buena parte del mundo de la cooperación, el discurso religioso se considera una intromisión ilegítima ${ }^{10}$. Por eso los mensajes de Manos Unidas y otras ONGD religiosas son a veces polisémicos, es decir, apuntan hacia una dirección u otra dependiendo del contexto (Bernstein, 2005).

\section{Conclusiones: poder, conflicto, isomorfismo y diversidad}

Los casos descritos ilustran cómo los conflictos internos han servido para modelar las presiones que empujan desde dentro y desde fuera a las ONGD hacia el isomorfismo. En la literatura sociológica sobre organizaciones, uno de los debates más interesantes es el relativo al isomorfismo, esto es, la tendencia de un conjunto de instituciones a presentar estructuras organizativas similares. En la introducción, hemos mencionado que mientras unos autores sostienen que las asociaciones de un campo organizativo tienden a parecerse (DiMaggio y Powell, 1983), otros autores matizan que, en ocasiones, algunos factores introducen heterogeneidad (Hambrick et al., 2005; Leiter, 2005).

Los casos estudiados nos permiten identificar factores que apuntan en direcciones opuestas: mientras unos parecen favorecer la homogeneidad entre

8. J. J. Omella (2005), La identidad de Caritas [en línea], <http://www.terra.es/personal/ cdlogdir/identidad\%20de\%20caritas.html> [consulta: 5 de febrero de 2007].

9. Lourdes AzORín (2007), «Humilde orgullo», Manos Unidas, 4-5.

10. A modo de ejemplo, el artículo 5 de los estatutos de la Coordinadora Andaluza de ONGD aclara que: «Aunque estas organizaciones estén inspiradas por una ideología política, religiosa o social específica, deberán estar en condiciones de garantizar en sus programas el carácter universal de la cooperación, desprovista de cualquier forma de proselitismo o discriminación». 
las ONGD, otros refuerzan la diversidad. Entre los factores que empujan a las ONGD a parecerse unas a otras destacan: la edad y el crecimiento organizativo (en participantes, delegaciones, actividades y fondos gestionados); las "modas», que hacen que las ONGD se imiten unas a otras en sus discursos, prácticas y estructuras organizativas, y, sobre todo, la existencia de un marco político, administrativo y legal común, que favorece una alta implicación de organizaciones de la sociedad civil en la provisión de determinados servicios y obliga a las ONGD a seguir unas mismas exigencias, muchas de las cuales implican contar con personal experto (profesionales) con una alta dedicación horaria (generalmente, contratados).

Por un lado, la edad y el crecimiento organizativo, favorecidos, entre otros factores, por el aumento de fondos públicos disponibles desde mediados de la década de 1990 hasta comienzos de la crisis de 2008, han obligado a las organizaciones a aumentar su eficacia (Blake, 2012; EDIS, 2012) y normalizar las actividades —o, utilizando la terminología de Mintzberg (1988), adoptar un modelo burocrático- . Así lo explicaba una trabajadora de una de las ONGD estudiadas:

En los últimos cuatro o cinco años, ha habido tal crecimiento que ha hecho necesario que haya profesionales. Se está moviendo una mayor cantidad de fondos propios, hay más delegaciones, vienen más voluntarios jóvenes... Y entonces, en ese sentido, ellos, a lo mejor, antes manejaban toda la información, o sea, estaban en Ayuda Humanitaria y sabían desde hace quince días que tal día iba a salir un contenedor de Ayuda Humanitaria, ¿̨no? Ahora a lo mejor lo saben porque leen los carteles. (Silvia, 37 años, empleada y gerente de la sede central de Madre Coraje, Jerez de la Frontera. Entrevista)

En efecto, una organización se burocratiza cuando las actividades pasan a ser coordinadas mediante normas que difuminan las relaciones entre los participantes y mediante procesos de selección de personal regulados e impersonales. Se produce una normalización de los procesos de trabajo, que pasan a ser programados, especificados y se crean instrucciones detalladas sobre cómo realizarlos. Los tres casos descritos han experimentado procesos de crecimiento y burocratización. A menudo, estos procesos generan un cambio en el perfil de los participantes (Blake, 2012), pues se incorpora a las organizaciones personas en las que el componente motivacional profesional y salarial adquiere mayores dimensiones. La presencia de este tipo de colaboradores puede generar tensiones internas (Piñón, 2010).

Por otro lado, existen numerosos casos de imitación, incluso «modas», en el sector de la cooperación (lenguajes, discursos, metodologías, prácticas). A modo de ejemplo de proceso mimético, citaremos el testimonio de una informante que contaba cómo se constituyó el área de Educación en la asociación Madre Coraje. Sencillamente, pretendían hacer lo mismo que las otras ONGD:

Y cuando nos planteamos la educación para el desarrollo, que ahí ya estaba yo. Me acuerdo perfectamente que, con Sergio, Antonio y Tomás, nos sentamos 
en una cafetería pensando que eso de la educación lo teníamos que hacer y no teníamos ni idea. Porque eran las tres patas, digamos, de la cooperación al desarrollo. Y recuerdo yo, allí en la cafetería, que cada uno buscaba, se estudiaba un arte y nos juntábamos: "A ver, ¿qué hacemos con esto? Pues hay una parte que se llama "educación formal", otra parte "educación", otra que se llama "comercio justo" o "incidencia política"». Y enterándonos de lo que era aquello. Y a partir de ahí, poner en marcha los proyectos. O sea, siempre se ha intentado estar un poco en la vanguardia de lo que es la cooperación al desarrollo ideológicamente. Siempre se ha intentado mucho. También ha habido parones, claro. No todos pueden ir al mismo ritmo. (Verónica, 40 años, responsable de Coordinación Territorial de Madre Coraje, Jerez de la Frontera. Entrevista)

Podemos decir que estos factores empujan al sector de las ONGD a adoptar estructuras burocráticas y profesionalizadas muy similares, así como a presentar un discurso con numerosos elementos provenientes de la gestión empresarial, lo cual desplaza a un segundo plano a otras actividades como la presión política (Carmel y Harlock, 2008).

$\mathrm{Al}$ igual que unos factores empujan hacia el isomorfismo, otros favorecen la heterogeneidad. Los que estimulan la diversidad son, especialmente, la presencia de figuras carismáticas y las relaciones estrechas con organizaciones e ideologías religiosas, políticas y sindicales ${ }^{11}$ —además de ser una organización pequeña y joven. El caso de Madre Coraje, por ejemplo, ilustra cómo un líder carismático puede frenar la tendencia isomórfica. Su fundador, Antonio Gómez, junto a sus seguidores, consiguió controlar y limitar el proceso de burocratización y profesionalización de la asociación. Esto se realizó, entre otras cosas, imponiendo un criterio determinado de selección de personal (primando, en palabras del presidente, la «actitud» frente a la «aptitud») o excluyendo a los empleados de los espacios de toma de decisiones.

Los casos de Paz y Solidaridad, perteneciente a Comisiones Obreras, y Manos Unidas, perteneciente a la Iglesia católica, ejemplifican cómo el control externo suele generar crisis internas en las ONGD, que se resuelven modificando las estructuras organizativas. Estas nuevas formas organizativas tienden a reproducir las estructuras de la organización externa, además de reforzar el control exterior. Paz y Solidaridad se quedó sin voluntariado cuando Comisiones Obreras decidió intensificar su control ${ }^{12}$. Manos Unidas, por su parte, tiene una estructura piramidal en cuya cúspide está la Comisión Permanente, un órgano de gobierno en el que siempre están presentes representantes de la Iglesia católica. Desde este punto de vista, el control externo limita el isomorfismo estructural. En el plano discursivo y de actividades, las relaciones con

11. Otros factores que favorecen la heterogeneidad, identificados en nuestro análisis, son el ámbito geográfico, las "culturas profesionales» (como las de los médicos, economistas o ingenieros) $y$, en mucha menor medida, la fórmula legal de la ONGD (como asociación, fundación, federación u otras).

12. De hecho, Paz y Solidaridad de Andalucía sólo puede entenderse como un apéndice, una parte de la estructura de Comisiones Obreras de Andalucía. 
organizaciones e ideologías religiosas, políticas y sindicales también favorecen la diversidad. Las relaciones con otras entidades amplían y redefinen las prácticas y los discursos del «desarrollo».

En ambos casos, el conflicto originado por el control externo sobre la ONGD sirvió para acotar el poder de los profesionales. La burocratización puede afectar a la estructura de poder de una organización, por eso, cuando ambas entidades crecieron en el entorno de la Iglesia católica y del sindicato Comisiones Obreras, éstos se apresuraron a controlar desde el exterior a sus ONGD. Existen diversas fórmulas para influir en una ONGD desde un ente externo: ubicar en la cúspide del organigrama a personas de la organización matriz, supervisar los ascensos, seguir métodos de reclutamiento de personal en los que se seleccionen personas fieles a la asociación, la presión ideológica a través de publicaciones y documentos o dotarla de recursos económicos, materiales y humanos.

A modo de conclusión, a través del marco político-legislativo y el sistema de financiación, las ONGD experimentan presiones hacia el isomorfismo. Es principalmente el contexto social y político, en el que cada vez más servicios sociales son provistos por entidades del Tercer Sector, el que empuja a las ONGD a profesionalizarse (Carmel y Harlock, 2008; Blake, 2012; EDIS, 2012). Además, la existencia de imitación entre distintas ONGD también favorece la homogeneidad en estructuras, actividades y discursos. Estos factores fomentan la burocratización y la profesionalización. Sin embargo, otros vectores, como las figuras carismáticas o las relaciones estrechas con entidades políticas, sindicales y religiosas, contribuyen a resistir las presiones isomórficas y preservar la diversidad en estructuras, prácticas y discursos. A través del conflicto, se moldea la forma en que el contexto influye en las organizaciones. Los conflictos internos experimentados en estas ONGD carismáticas o con alto control externo han servido para frenar las presiones del sector y preservar la diversidad: mantener culturas organizativas, visiones y prácticas «asistencialistas» que atraen a una base social determinada; por ejemplo: se conservan discursos que combinan el «desarrollo» y el sindicalismo o la ayuda y la fe católica.

Los casos nos enseñan cómo el conflicto no sólo es algo consustancial a las organizaciones, sino que puede ser adaptativo en términos ecológicos, lo cual favorece la diversidad y configura una identidad organizativa compartida. La diversidad puede ser crucial si las ONGD aspiran a mantener su legitimidad social y a preservar algunas de las propiedades que se les suelen asignar, como la flexibilidad y la capacidad de innovación.

\section{Referencias bibliográficas}

Aguirre, Ángel (2004). La cultura de las organizaciones. Barcelona: Ariel.

BERNSTEIN, Erika (2005). «Transcending politics through the Kingdom of God and Free Markets: A case study of religious NGOs in Zimbabwe». En: IGOE, Jim y KeTSAll, Tim (eds.) (2005). Between a rock and a hard place: African NGOs, donors and the State. Durham, Carolina del Norte: Carolina Academic Press, 63-90. 
Blake, J. (2012). Professionalism and the third sector. Comunicación presentada en el Annual Meeting of the ISTR $10^{\text {th }}$ Internacional Conference.

CARMEL, Emma y HarlocK, Jenny (2008). «Instituting the "third sector" as a governable terrain: Partnership, procurement and performance». Policy \& Politics, 36 (2), 155-171. <http://dx.doi.org/10.1332/030557308783995017>

DimagGio, P. J. y POWELL, Walter W. (1983). «The iron cage revisited: Institutional isomorphism and collective rationality in organizational fields». American Sociological Review, 48, 147-160.

<http://dx.doi.org/10.2307/2095101>

DURKHEIM, Émile (1995). Las reglas del método sociológico. Barcelona: Atalaya, 1895.

EDIS (2012). Anuario del Tercer Sector de Acción Social en España. Madrid: Fundación Luis Vives y Caja Madrid.

«En torno a la crisis de Manos Unidas». Revista de Fomento Social, 55 (2000), 311-339.

ESCARTín, Pedro (2009). Declararon la guerra al hambre: Cincuenta años en la vida de Manos Unidas. Madrid: Manos Unidas.

Hambrick, D. C.; Finkelstein, Sydney; Cho, Theresa S. y Jackson, Eric M. (2005). "Isomorphism in reverse: Institutional theory as an explanation for recent increases in intraindustry heterogeneity and managerial discretion». Research in Organizational Behavior, 26, 307-350.

<http://dx.doi.org/10.1016/S0191-3085(04)26008-7>

HofSTEDE, Geert (1999). Cultura y organizaciones. Madrid: Alianza.

LeITER, J. (2005). «Structural Isomorphism in Australian Non-profit Organizations». Voluntas, 16 (1), 1-31. <http://dx.doi.org/10.1007/s11266-005-3230-1>

MAYNTZ, Renate (1996). Sociología de la organización. Madrid: Alianza.

Memoria 1991-2000. Jerez de la Frontera: Asociación Madre Coraje, 2000.

Memoria 2004. Jerez de la Frontera: Asociación Madre Coraje, 2004.

Memoria 2009. Jerez de la Frontera: Madre Coraje, 2009.

Memoria 2009. Madrid: Fundación Paz y Solidaridad Serafín Aliaga, 2009.

Michels, Robert (1996). Los partidos políticos: Un estudio sociológico de las tendencias oligárquicas de la democracia moderna. Buenos Aires: Amorrortu.

MinTZBERG, Henry (1988). La estructuración de las organizaciones. Barcelona: Ariel.

NúNEZE, Maite y SANTOS, Gracia (2004). «Otros agentes: Los sindicatos». En: BENGOA, Juana y CALLEJÓN, María Eugenia (coords.). La palabra empeñada: Los objetivos 2015 y la lucha contra la pobreza. Madrid: Catarata, 177-185.

PARSONS, Talcott (1988). El sistema social. Madrid: Alianza, 1952.

PIÑón, Josefina (2010). «Empleo y trabajo voluntario en el sector de la intervención social y de cooperación al desarrollo». Sociología del Trabajo, 69 (verano), 49-71.

RaWls, Anne W. (2008). "Harold Garfinkel, Ethnomethodology and Workplace Studies». Organization Studies, 29 (5), 701-732.

<http://dx.doi.org/10.1177/0170840608088768>

SCHEIN, Edgar H. (1986). Organizational culture and leadership. San Francisco y Londres: Jossey-Bass Publishers.

SCHWARTZMAN, H. B. (1993). Ethnography in organizations. Newbury Park / Londres / Nueva Delhi: Sage Publications.

Sсотт, W. Richard (2001). Institutions and organizations. Thousand Oaks / Londres / Nueva Delhi: Sage Publications.

VIDAL, Pilar (1997). Una historia de solidaridad: Manos Unidas y la ayuda al desarrollo. Madrid: Manos Unidas. 